\title{
Variability: A X-ray ruler for the AGN structure model
}

\author{
M. Guainazzi \\ XMM-Newton Science Operations Centre, European Space Astronomy Centre of ESA, Camino bajo del Castillo, \\ s/n Urbanización Villafranca del Castillo Villanueva de la Cañada, 28692 Madrid, Spain
}

\begin{abstract}
Validating our understanding of the innermost structure of Active Galactic Nuclei (AGN) would require resolving sub-parsec scales. Lacking adequate direct imaging, X-ray astronomy can still contribute to this undertaking through the study of spectral variability on time-scales from days to years. This bears information on the location of gaseous and dusty systems in the innermost regions around the accreting supermassive black hole. In this paper I discuss the application of this concept in two specific contexts: a) "fast" column density variations in heavily obscured AGN; b) reverberation of optically-thick reprocessing. These results lend support to a scenario where obscuration and optically thick reprocessing are due to a variety of different systems, ranging from the Broad Line Regions to a clumpy structure extended on larger scales up to hundreds of parsecs.
\end{abstract}

\section{INTRODUCTION}

Validating our understanding of the innermost structure of Active Galactic Nuclei (AGN) requires resolving subparsec scales. This is currently possible only at radio wavelengths. For all the other energy domains (and, most notably, for radio-quiet AGN) one id compelled to use indirect techniques to pinpoint the location of the gaseous and dusty systems surrounding the accreting supermassive black hole.

Recently, mid-IR interferometry has allowed for the first time to effectively "image" the dusty component of the parsec-scale torus ${ }^{1}$. In the three obscured AGN for which such measurements have been possible so far (NGC 1068, the Circinus Galaxy, and NGC 4151), the data are consistent with a two-temperature distribution of dust, with most the of the obscuration occurring on scales smaller than 1 pc $[6,14,44]$. In Type 1 AGN, near-IR studies $[15,16]$ indicate that the size of the innermost torus is primarily driven by the $\propto L^{1 / 2}$ dust sublimation radius. Once this dependence is removed, the dusty structure is more compact in more luminous objects. Covering fraction is generally large, often exceeding $80 \%$.

Other techniques to estimate spatial scaling in AGN make use of time variability. Reverberation mapping of optical-UV lines to changes of the ionising continuum $[5,29,31]$ has been used for almost three decades to measure the mean light-travel time across the Broad Line Region (BLR) clouds [30]. While the estimate of the virial mass from the emission-line width and the measured time delay is dependent on an unknown geometrical factor depending on the structure, orientation and kinematics of the BLRs - derived masses of the supermassive black hole have proved to be so robust, that they could be used to calibrate the cosmic distance ladder [46].

In this paper, I will focus on X-ray based methods to determine the location of gaseous and dusty systems responsible for obscuration and/or optically thick

\footnotetext{
${ }^{1}$ We use this rather inaccurate nomenclature primarily for historical reasons. As discussed in $\$ 4$, X-ray results indicate that the absorbing/reprocessing material surrounding the central engine is most likely not arranged in the shape of a compact and homogeneous torus.
}

reprocessing of the AGN high-energy primary emission. These methods rely on measuring either the smallest possible variability timescales of the column density in heavily obscured AGN ( $\$ 2)$, or the response to changes of the primary continuum in spectral regions dominated by reprocessing $(\$ 3)$. In a few cases, extended emission on the arcsecond $(\simeq 100 \mathrm{pc})$ scale allows direct imaging of the X-ray torus, as well as of the X-ray Extended Narrow Line Regions. The latter system will not be discussed in this paper. Readers are referred to a vast recent literature on this field $[2,40,45]$.

\section{FAST BLR X-RAY OBSCURING CLOUDS?}

NGC 1365 is a Seyfert 2 Galaxy extensively monitored over the whole recent history of X-ray astronomy. The main reason behind this interest is related to its intriguing spectral variability in. Above $3 \mathrm{keV}$, NGC 1365 has been observed to display two distinct spectral "states": a) a power-law photoelectrically obscured by a Comptonthin column density of the order of $10^{23} \mathrm{~cm}^{-1}$ [18]; b) a "reflection-dominated" spectrum, characterised by an extremely flat continuum (photon index, $\Gamma, \simeq 0$ ), a strong iron $\mathrm{K}_{\alpha}$ fluorescent line from neutral or mildly ionised iron, and a deep iron photo-absorption edge [37]. During a monitoring campaign in April 2006, NGC 1365 was caught oscillating between these two states on a timescale of two days [35]. If one interprets the "reflectiondominated" state as the reprocessing echo of an otherwise invisible primary, and attributes the disappearance of AGN signatures to obscuration by Compton-thick clouds (hence the alternative nomenclature of "Compton-thick" given to these "reflection-dominated" states), such occultation episodes provide a unique tool to constrain the size and location of the obscuring clouds, as well as that of the X-ray corona.

Assuming occultation by a single cloud in Keplerian motion around a supermassive black hole, the distance between the X-ray source and the cloud can be expressed as:

$$
d \sim 600 t_{100}^{2} n_{10}^{2} N_{H, 24}^{-2} R_{S}
$$

where $t_{100}$ is the variability timescale in units of 100 seconds, $n_{10}$ is the cloud density in units of $10^{10} \mathrm{~cm}^{10}$, 
$N_{H, 24}$ the column density in units of $10^{24} \mathrm{~cm}^{-2}$ and $R_{S}$ is in units of Schwarzschild radii. Independent constraints on $n$ can be obtained by the exact ionisation state of the iron fluorescent line, allowing to solve the above equation for all its unknowns. Coupling these results with a constraint on the Keplerian velocity, $v_{K}$, obtained from a measurement of the width of the iron line profile, one can add a constraint on the size of the X-ray corona.

Obscuration events similar to what observed in NGC 1365 have been observed so far on a handful of heavily obscured AGN, covering a wide range of time-scales (due to the sparse nature of the observations available, and the serendipitous nature of some of these discoveries): UGC 4203 ("The Phoenix Galaxy"; [13,34]), Mkn 348 [42], NGC 3227 [19], NGC 4388 [8], NGC 4151 [33], NGC 7582 [4], Centaurus A [39]. While each source would deserve a detailed discussion in its own, in most cases the properties of the absorbing clouds derived from these events share common properties (see, e.g., [21]):

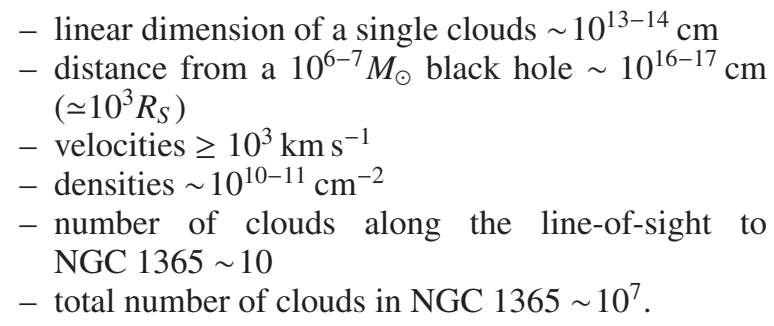

It is difficult to extrapolate these results to the overall population of obscured AGN in the local Universe. By selection, the galaxies where these extreme X-ray variability events have been seen are potentially "extreme". Moderate variability of the obscuring column density is ubiquitous [36] whenever a sufficiently long temporal baseline is available. On the other hand, X-ray obscuration "state transitions" (the "changing-look" AGN; [25]) are rare on a human-life time scale, possibly occurring once in a century for each individual object [11] except in extreme sources such as NGC 1365.

NGC 1365, thanks to its extremely fast variability and consequently intense monitoring, has also allowed the best test so far of the assumption of simple spherical clouds in Keplerian motion. A long observation with Suzaku unveiled two episodes of extremely fast (about a few thousands seconds) decrease in the hard energy $(E>2 \mathrm{keV})$ flux [21]. After each of these episodes, the absorber column density decreases, while its covering fraction increases. The absorbing cloud is therefore consistent with a cometary shape, with opening angles $\sim 1-5^{\circ}$ and a column density gradient of about one order of magnitude (from $\sim 10^{23}$ to $\sim 10^{22} \mathrm{~cm}^{-2}$ ) from head to tail. A similar phenomenology was unveiled in a long XMMNewton observation of Mkn 766 [38], and interpreted as due to "head-on" cometary clouds.

\section{X-RAY REPROCESSING REVERBERATION}

Reverberation of the high-energy AGN primary emission by optically thick gas is the primary method to estimate the distance to the "torus" (or a lower limit thereof)

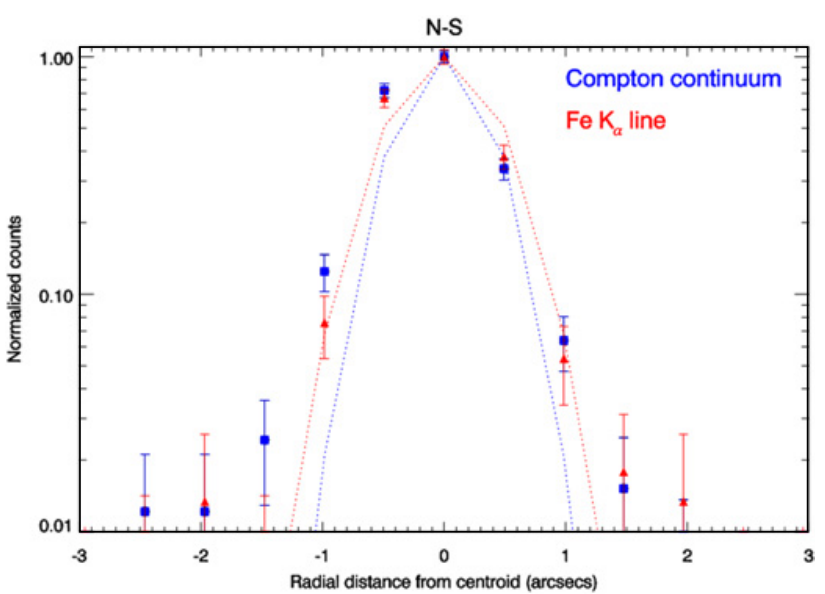

Figure 1. Background-subtracted profiles of the $0^{\text {th }}$ order image of the Chandra ACIS-s/HETG observation of Markarian 3 in the 3.5-5.5 keV (blue) and the 6-7 keV (red) energy bands. The dotted lines are the corresponding PSF profiles.

in X-rays. In Compton-thin sources, the Comptonreflection continuum is outshone by the transmitted primary emission. Even in these cases, however, $\mathrm{X}$-ray torus reverberation is possible by using the $\mathrm{Fe} \mathrm{K}_{\alpha}$ fluorescent iron line. Compton continuum reverberation is possible only in Compton-thick sources, provided that a light curve with sufficient statistics is available above the photoelectric and Compton-scattering cut-off, and that the column density is not so high to significantly suppress also the transmitted component (as it occurs, e.g. in the best studied Compton-thick AGN of the local Universe, NGC 1068 [24]). These measurements are, however, rare, because they require regular monitoring campaigns on a wide X-ray energy range, and on timescales of the order of weeks to years.

In most of cases, such campaign detect no variability of the reprocessed spectral components. The corresponding lower limit on the distance to the inner side of the torus are $2.5 \mathrm{pc}$ in NGC 5506 (where the size of the BLR is 10 light-days; [10]), $3.2 \mathrm{pc}$ in NGC 2992 [47], and $\geq 30-50$ pc in NGC 4945 [22]. In the last object, a Suzaku campaign, coupled with historical Chandra and XMMNewton data, unveiled that spectral components associated to reprocessing are constant within $<10 \%$, while the Swift/BAT light curve, probing the intrinsic AGN emission, exhibits variability by a factor of 2 on time-scales of the order of one year. At the distance of NGC 4945, the repreocessor should cover 2-3 arcseconds if on the plane of the sky. Indeed, high-resolution imaging with Chandra in a narrow band dominated by the iron $\mathrm{K}_{\alpha}$ shows a flattened extended structure on a projected scale $\simeq 200 \times 100$ pc. Iron band reprocessing on a similar spatial scale is known in NGC 1068 as well [45].

A particularly intriguing case is Markarian 3. Similarly to NGC 1068 and NGC 4945, Chandra images suggest a reprocessing feature in Markarian 3 extending up to $\simeq 300 \mathrm{pc}$ [12] perpendicular to the X-ray Extended Narrow Line Regions [40]. Tentatively, this feature is more prominent in the Compton-reflection continuum (3.5$5.5 \mathrm{keV}$ energy band) than in the $\mathrm{Fe} \mathrm{K}_{\alpha}$ line (Fig. 1). This 
finding is difficult to reconcile with spectral variability results. The Compton-reflection flux in Markarian 3 is variable on time-scales as short as $\simeq 2$ months. A crosscorrelation with the Swift/BAT light curve indicates a delayed response to changes of the primary continuum on time-scales longer them 3 years. A simple geometrical model where the reprocessor is approximated by a simple slab requires an opening angle $\geq 71^{\circ}$, at odds with the discovery of the extended reprocessing feature. On the other hand, the lack of correlation between the Compton flux and the intensity of the iron $\mathrm{K}_{\alpha}$ line is consistent with their possible different morphology.

Variability of reprocessing spectral components has been so far elusive. Variability by about $25 \%$ of the iron $\mathrm{K}_{\alpha}$ line on timescales of about 1 years was reported by different authors in NGC $4151[28,41,43,48]$, suggesting that the optically thick reprocessing material in this source is at about $0.03 \mathrm{pc}$ from the nucleus. Markarian 3 is the only obscured AGN in the local universe, where evidence has been found of reprocessing by optically thick gas on scales from a fraction of to several hundreds parsecs.

\section{IMPLICATIONS FOR THE AGN STRUCTURE MODEL}

One possible explanation for these apparently contradicting results is that the absorber is clumpy. Qualitatively, reflection on different spatial scales require that absorption-free line-of-sights to the active nucleus must exist for clouds located at various distances. The case for a clumpy torus was originally advocated by Krolik \& Begelman (1998), and more recently fully developed in the calculations by Nenkova et al. (2002, 2008). Evidence for the torus clumpiness are mainly yielded by the comparison between torus models and multiwavelength Spectral Energy Distributions. A compact, pc-scale torus can hardly reproduce the multi-temperature IR emission [32]. Large-scale dusty torii can reproduce the observations [9], but are nowadays disfavoured given the IR interferometric evidence of very compact warm dust structures surrounding nearby obscured AGN (cf. §1).

Recently [7] proposed that the complex structure of the absorber/reflecting matter in the AGN environment should lead us to a revision of our interpretation of the AGN Unification Scenario. Its strictest formulation postulates that the orientation with respect to an azimuthallysymmetric obscuring structure is the only driving factor in the classification of an AGN [1]. Elitzur suggests that this view should be replaced by a probabilistic interpretation, whereby the classification of a radio-quiet AGN would depend on the covering factor distribution of the obscuring matter. "Type 2" would be more likely identified in AGN surrounded by matter with a higher covering factor. A least extreme view, where the Poissonian fluctuations in the number of optically-thick clouds on scales comparable or lower then the BLR is superposed to the standard formulation of the Unified Scenario is discussed by [23] and [3].

It can hardly be overestimated the role that ALMA will play in deepening our understanding of dust structures in the innermost regions of AGN with its superb spatial resolution. Direct imaging of the torus structure will be possible on scales as small as a few parsecs in nearby obscured AGN [20].

\section{References}

[1] Antonucci R., ARA\&A, 31 (1993) 473

[2] Bianchi S., Guainazzi M., Chiaberge M., A\&A, 448 (2006) 499

[3] Bianchi S., Maiolino R., Risaliti G., AdAST, 2012 (2012) 17

[4] Bianchi S., Piconcelli E., Chiaberge M., JiménezBailón E., Matt G., Fiore F., ApJ, 695 (2009) 781

[5] Blandford R.D. \& McKee C.F., ApJ, 255 (1982) 419

[6] Burtscher L., Jaffe W., Röttgering H., Meisenheimer K., Tristram K.R.W., MNRAS, 394 (2009) 1325

[7] Elitzur M., ApJ, 747 (2012) L33

[8] Elvis M., Risaliti G., Nicastro F., Miller J.M., Fiore F., Puccetti S., ApJ, 615 (2004) L25

[9] Granato G.L., Danese L., MNRAS, 268 (1994) 235

[10] Guainazzi M., Bianchi S., Matt G., Dadina M., Kaastra J., Malzac J., Risaliti G., MNRAS, 406 (2010) 2013

[11] Guainazzi M., Fabian A.C., Iwasawa K., Matt G., Fiore F., MNRAS, 356 (2005) 295

[12] Guainazzi M., La Parola V., Miniutti G., Segreto A., Longinotti A.L., arXiv.1209.0706 (2012)

[13] Guainazzi M., Matt G., Fiore F., Perola G.C., A\&A, 388 (2002) 787

[14] Jaffe W., Meisenheimer K., Röttgering H.J.A., et al., Nat, 429 (2004) 47

[15] Kishimoto M., Hönig S.F., Antonucci R., et al., A\&A 536 (2011a) 78

[16] Kishimoto M., Hönig S.F., Antonucci R., et al., A\&A, 527 (2011b) 121

[17] Krolik J.H., Begelman M.C., ApJ, 329 (1998) 702

[18] Iyomoto N., Makishima K., Fukazawa Y., Tashiro M., Ishisaki Y., PASJ, 49 (1997) 425

[19] Lamer G., Uttley P., McHardy I.M., MNRAS, 342 (2003) L41

[20] Maiolino R., NewAR, 52 (2008) 339

[21] Maiolino R., Risaliti G., Salvati M., et al., A\&A, 517 (2010) 47

[22] Marinucci A., Risaliti G., Wang Junfeng, et al., MNRAS, 423 (2012) L6

[23] Matt G., A\&A, 355 (2000) L13

[24] Matt G., et al., A\&A, 341 (1999) L39

[25] Matt G., Guainazzi M., Maiolino R., MNRAS, 342 (2003) 422

[26] Nenkova M., Ivezić Z., Elitzur M., ApJ, 570 (2002) L9

[27] Nenkova M., Sirocky M.M., Nikutta R., Ivezić Z., Elitzur M., ApJ, 685 (2008) 160

[28] Perola G.C., Piro L., Altamore A., et al., ApJ, 306 (1986) 508

[29] Peterson B., PASP, 105 (1993) 247

[30] Peterson B.M., Bentz M.C., NewAR, 50 (2006) 796

[31] Peterson B.M., Ferrarese L., Gilbert K.M., et al., ApJ 613 (2004) 682

[32] Pier E.A., Krolik J.H., ApJ 399 (1992) L23 
[33] Puccetti S., Fiore F., d'Elia V., et al., MNRAS, 377 (2007) 607

[34] Risaliti G., Elvis M., Bianchi S., Matt G., MNRAS, 406 (2010) 20

[35] Risaliti G., Elvis M., Fabbiano G., Baldi A., Zezas A., Salvati M., ApJ, 659 (2007) L111

[36] Risaliti G., Elvis M., Nicastro F., ApJ, 571 (2002) 234

[37] Risaliti G., Maiolino R., Bassani L., A\&A, 356 (2000) 33

[38] Risaliti G., Nardini E., Salvati M., et al., MNRAS, 410 (2011) 1027

[39] Rivers E., Markowitz A., Rothschild R., ApJ, 742 (2011) 29

[40] Sako M., Kahn S.M., Paerels F., Liedahl D.A., ApJL, 543 (2000) L115
[41] Schurch N.J., Warwick R.S., Griffiths R.E., Sembay S., MNRAS, 345 (2003) 423

[42] Smith D.A., Georgantopoulous I., Warwick R.S., ApJ, 550 (2001) 365

[43] Takahashi K., Inoue H., Dotani T., PASJ, 54 (2002) 373

[44] Tristram K.R.W., Meisenheimer K., Jaffe W., et al., A\&A, 474 (2007) 837

[45] Young A.J., Wilson A.S., Shopbell P.L., ApJ, 556 (2001) 6

[46] Watson D., Denney K.D., Vestergaard M., Davis T.M., ApJ, 640 (2011) 49

[47] Weaver K.A., Nousek J., Yaqoob T., Mushotzky R. F., Makino F., Otani C., ApJ, 458 (1996) 160

[48] Zdziarski A.A., Leighly K.M., Matsuoka M., Cappi M., Mihara T., ApJ, 573 (2002) 505 